Pharmacogn. Mag.

\title{
Decalepis hamiltonii and its Bioactive Compounds Protects Isoproterenol-Induced Myocardial Oxidative Stress in Rats
}

\author{
Anupama Sindhaghatta Kariyappa, Sowbhagya Ramachandregowda', Santosh Anand', Bhagyalakshmi Dundaiah, \\ Mamatha Madhugiri Gopinath, Ravikiran Tekupalli
}

Department of Biotechnology, Jnanabharathi Campus, Bangalore University, 'Ramaiah College of Arts Science and Commerce, Bengaluru, Karnataka, India

Submitted: $19-12-2018$

Revised: 31-01-2019

Published: 26-08-2019

\begin{abstract}
Background: Decalepis hamiltonii $(D h)$, a climbing shrub belonging to the family Apocynaceae, is consumed for its health-promoting properties. Objective: The present study was designed to evaluate the cardioprotective potential of $D h$ extract and its bioactive compounds on region-specific responses against isoproterenol (ISO)-induced myocardial injury in male Wistar rats. Materials and Methods: Rats were orally supplemented with ellagic acid (50 mg/kg body weight [BW]), 4-hydroxy isophthalic acid (30 mg/kg BW), and Dh extract (100 mg/kg BW) for 30 days, and they were subsequently administered (intraperitoneally) with ISO (150 mg/kg BW) for the last 2 days. Results: ISO-treated rats showed a significant increase in serum lipid profile, markers of cardiac damage, and decreased antioxidant status. Supplemented groups showed improved lipid profile, reduced serum marker enzymes, and enhanced antioxidant status in ISO-administered rats. Histopathological studies further confirmed the protective effect of $D h$ extract against ISO-induced myocardial infarction (MI). Conclusion: Our results demonstrated that $D h$ extract and its compounds efficiently ameliorated ISO-induced $\mathrm{MI}$ in rats.

Key words: Decalepis hamiltonii, isoproterenol, myocardial infarction, oxidative stress, serum lipid profile

\section{SUMMARY}

- Effect of Decalepis hamiltonii (Dh) extract and its bioactive compounds against isoproterenol (ISO)-induced myocardial infarction was investigated

- The ISO-treated rats showed increased oxidative stress and myocardial damage as evidenced by different biochemical parameters and histoarchitecture

- Pretreatment of Dh extract and its compounds diminished the ISO-induced oxidative stress and improved the antioxidant status

- Our results confirm that Dh and its compounds offer cardioprotective activity.
\end{abstract}

Abbreviations used: Dh: Decalepis hamiltonii; EA: Ellagic acid; 4-HIA: 4-hydroxy isophthalic acid; ISO: Isoproterenol; MI: Myocardial infarction; LV: Left ventricle; RV: Right ventricle; SOD: Superoxide dismutase; CAT: Catalase; GPx: Glutathione peroxidase.

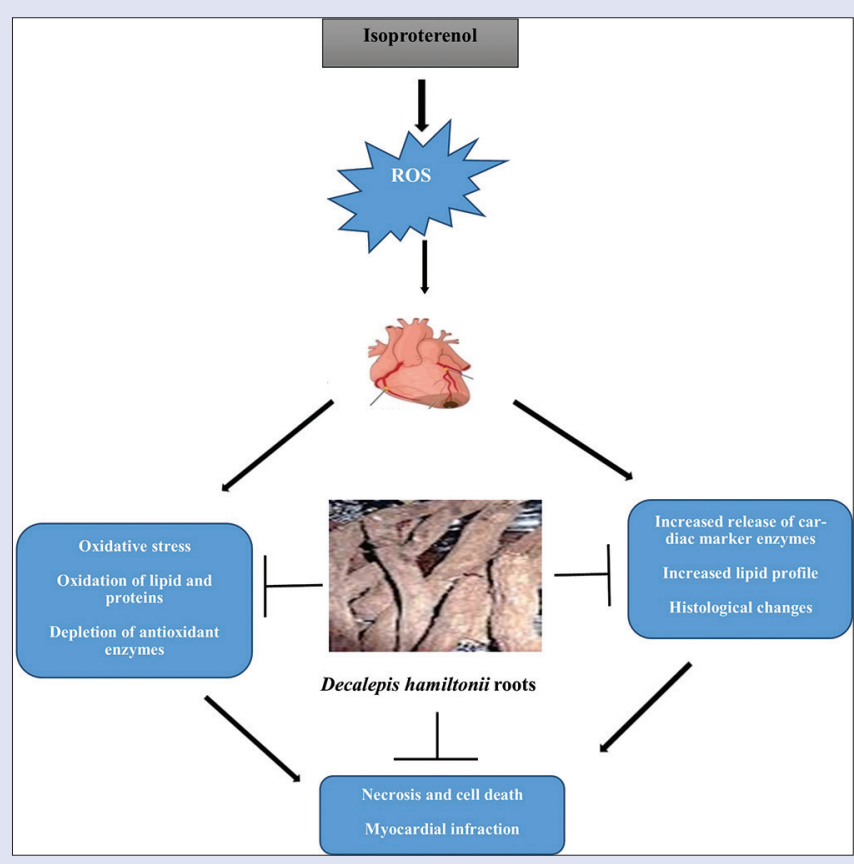

Correspondence:

Dr. Ravikiran Tekupalli, Department of Biotechnology, Jnanabharathi Campus, Bangalore University, Bengaluru - 560 056, Karnataka, India. E-mail: ravikiran@bub.ernet.in DOI: $10.4103 / p m . p m \_642 \_18$
Access this article online Website: www.phcog.com Quick Response Code:

\section{INTRODUCTION}

Myocardial infarction (MI) is one of the leading causes of mortality and morbidity, worldwide. ${ }^{[1]}$ It occurs as a consequence of the imbalance between myocardial oxygen demand and coronary blood supply. It is characterized by structural and functional modifications in the myocardium, resulting in contractile dysfunction, ventricular dilatation, and subsequent heart failure. ${ }^{[2]}$

Isoproterenol (ISO) is a synthetic catecholamine and $\beta$-adrenergic agonist that causes severe stress in the myocardium, resulting in infarct-like necrosis of the heart muscle. The pathophysiological and morphological changes observed in ISO-administered rats are similar to MI in humans. ${ }^{[3]}$ Further, it undergoes autoxidation generating highly toxic reactive oxygen species (ROS) that stimulate lipid peroxidation, leading to both structural

and functional myocardial injury. Therefore, ISO-induced myocardial injury serves as a well-standardized model for human MI to study the beneficial effects of drugs on cardiac function. ${ }^{[4]}$

This is an open access journal, and articles are distributed under the terms of the Creative Commons Attribution-NonCommercial-ShareAlike 4.0 License, which allows others to remix, tweak, and build upon the work non-commercially, as long as appropriate credit is given and the new creations are licensed under the identical terms.

For reprints contact: reprints@medknow.com

Cite this article as: Kariyappa AS, Ramachandregowda S, Anand S, Dundaiah B, Gopinath MM, Tekupalli R. Decalepis hamiltonii and its bioactive compounds protects isoproterenol-induced myocardial oxidative stress in rats. Phcog Mag 2019; 15:S320-7. 
In recent years, the therapeutic potential of medicinal plants has gained interest as antioxidants in reducing free radical-induced tissue injury. Among conventional herbs, Decalepis hamiltonii (Dh) (Apocynaceae) has been used as a common ingredient in many of the Ayurvedic preparations. The roots possess potent antioxidant properties, which could be responsible for various health benefits. ${ }^{[5]}$ The aqueous root extract is a cocktail of many polyphenolic compounds which include ellagic acid (EA), 4-hydroxy isophthalic acid (4-HIA), 14-aminotetradecanoic acid, 4-(1-hydroxy-1-methyl ethyl)-1-methyl-1,2-cyclohexanediol, 2-hydroxymethyl-3-methoxybenzaldehyde, and 2,4, trihydroxybicyclo -octan-3-one. ${ }^{[6]}$ Studies have shown that $D h$ root extract exhibits antiatherosclerotic, antidiabetic, hepatoprotective, and neuroprotective activities. ${ }^{[7]}$ We also reported that EA and 4-HIA are the major phenolic compounds in the $D h$ extract, which exhibited protective effects against peroxyl-induced oxidative stress in the rat erythrocytes. However, to our knowledge, limited reports are available regarding the cardioprotective effects of $D h$ extract and its constituents.

Therefore, the present study was hypothesized to investigate the cardioprotective effects of $D h$ extract and its compounds in ameliorating ISO-induced MI in rats. The hypothesis was examined by assessing the biochemical markers of cardiac injury as well as antioxidant enzyme activities and markers of oxidative stress in the left ventricle (LV) and right ventricle (RV) regions of the heart. Further, histopathological studies were also carried out to understand the cardioprotective effects of $D h$ extract.

\section{MATERIALS AND METHODS}

\section{Chemicals}

EA, 4-HIA, epinephrine, reduced glutathione (GSH), glutathione reductase, thiobarbituric acid (TBA), t-butyl hydroperoxide, guanidine hydrochloride, reduced nicotinamide dinucleotide phosphate (NADPH), reduced nicotinamide adenine dinucleotide, nitro blue tetrazolium (NBT), and 5,5'-dithio-bis-(2-nitrobenzoic acid) (DTNB) were procured from Sigma (St. Louis, MO, USA). All other chemicals and solvents were of analytical grade.

\section{Preparation of aqueous root extract}

Dh roots were collected from Savanadurga Forest, Magadi Taluk, Ramanagara District, India. The plant material was identified at the Department of Botany, Bangalore University, Bengaluru. A voucher specimen was deposited in the herbarium (BUB, No. 2223). The aqueous root extract was prepared according to the method of Ravikiran et al. ${ }^{[8]}$ Briefly, the outer fleshy part was separated from the inner woody core using a scalpel, dried at $40^{\circ} \mathrm{C}$, and finely powdered. The aqueous extract of the outer fleshy part (100 g) was prepared by dissolving the powder in $1 \mathrm{~L}$ of warm water $\left(50^{\circ} \mathrm{C}\right)$ and allowing it to stand for $24 \mathrm{~h}$ and filtered through Whatman no. 1 paper. The filtrate was lyophilized, weighed $(17.5 \mathrm{~g})$, and stored at $4^{\circ} \mathrm{C}$ until further use.

\section{Animal maintenance}

Adult male Wistar rats weighing 200-220 g were obtained from the Central Animal Facility, IISc, Bengaluru. The animals were placed three per cage in polypropylene cages fitted with stainless steel wire-mesh bottoms, maintained at a temperature of $28^{\circ} \mathrm{C} \pm 1{ }^{\circ} \mathrm{C}$, relative humidity of $77 \% \pm 1 \%$, and under 12 -h dark and light cycle. All animals had free access to food (Amruth Feeds, Bengaluru) and tap water ad libitum. The study protocol was approved by the Institutional Animal Ethical Committee (IAEC) (BUB/IAEC/TRK/05/2015), Department of Zoology, Bangalore University, Bengaluru, and complied with the guidelines of the Committee for the Purpose of control and Supervision of Experiments on Animals.

\section{Dosage fixation}

Different doses of EA $(25,50,75$, and $100 \mathrm{mg} / \mathrm{kg}$ body weight [BW]), 4-HIA $(10,20,30,40$, and $50 \mathrm{mg} / \mathrm{kg} \mathrm{BW})$, and $\mathrm{Dh}$ extract $(50,100,150$, and $200 \mathrm{mg} / \mathrm{kg} \mathrm{BW}$ ) were screened to determine the dose-dependent effect in ISO-induced myocardial-infarcted rats. The effective doses were assessed based on the activities of lactate dehydrogenase (LDH), creatine kinase-MB (CK-MB), levels of antioxidant enzymes, and markers of oxidative stress. EA (50 mg/kg BW), 4-HIA (30 mg/kg BW), and Dh extract $(100 \mathrm{mg} / \mathrm{kg} \mathrm{BW})$ were found to be most effective in reducing the biochemical markers and enhancing the antioxidant status. Hence, these doses were fixed for further studies.

\section{Experimental design}

A total of 40 rats were randomly divided into eight groups.

- Group I: Normal controls rats (normal saline p.o)

- Group II: ISO-treated

- Group III, IV, and V: Rats pretreated with EA, 4-HIA, and Dh extract, respectively

- $\quad$ Group: VI, VII, and VIII: Rats pretreated with EA, 4-HIA, and Dh extract, respectively, with ISO.

EA, 4-HIA, and $D h$ extract were prepared in normal saline and administered at a dosage of $50 \mathrm{mg} / \mathrm{kg}$ BW (EA), $30 \mathrm{mg} / \mathrm{kg} \mathrm{BW} \mathrm{(4-HIA),}$ and $100 \mathrm{mg} / \mathrm{kg} \mathrm{BW}$ (Dh extract), respectively, using an intragastric tube for 30 days. Group II rats were given normal saline for 27 days and injected intraperitoneally (i.p.) with ISO at a dosage of $150 \mathrm{mg} / / \mathrm{kg} \mathrm{BW}$ consecutively on the $28^{\text {th }}$ and $29^{\text {th }}$ day at an interval of 24 h. ${ }^{[9]}$ Group VI, VII, and VIII received the compounds and $D h$ extract for 30 days followed by ISO (150 mg/kg BW i.p.) at an interval of $24 \mathrm{~h}$ for the last 2 days.

\section{Tissue preparation}

After completion of the experimental protocol, the animals were sacrificed on the $30^{\text {th }}$ day under diethyl ether anesthesia followed by cervical dislocation. ${ }^{[10]}$ The heart was excised and LV and RV regions were separated, washed with ice-cold saline, and weighed. The tissue homogenates were prepared in phosphate buffer $(50 \mathrm{mM}, \mathrm{pH} 7.4)$ and centrifuged at $2000 \times g$ for $10 \mathrm{~min}$ (Superspin RV/FM, Plasto Crafts). The homogenates were used for the estimation of malondialdehyde (MDA), superoxide radical (SOR), and thiols (SHs). The remaining homogenate was centrifuged at $2000 \times \mathrm{g} \mathrm{rpm}$ at $4^{\circ} \mathrm{C}$ for $10 \mathrm{~min}$. The supernatant obtained was used for antioxidant enzyme assays and protein carbonyl (PC) estimation.

\section{Biochemical assays in the serum}

Blood was collected by cardiac puncture under ether anesthesia and allowed to clot for $30 \mathrm{~min}$ at RT. The serum was separated by centrifugation at $2500 \mathrm{rpm}$ for $15 \mathrm{~min}$. Total cholesterol (TC), triglycerides (TGs), high-density lipoprotein (HDL), low-density lipoprotein (LDL) levels as well as the activities of $\mathrm{LDH}$ and CK-MB were determined in the serum using commercially available kits (Code No. 731 LS102-25, Span Diagnostics Ltd., India).

\section{Measurement of lipid peroxidation}

MDA content was measured according to the procedure of Ohkawa et al. ${ }^{[1]}$ using 1,1,3,3-tetramethoxypropane as the standard. Briefly, $100 \mu \mathrm{L}$ of homogenate was added to $200 \mu \mathrm{L}$ of $8.1 \%$ Sodium dodecyl sulphate (SDS); $1.5 \mathrm{~mL}$ of $20 \%$ acetic acid and $1.5 \mathrm{~mL}$ of $0.8 \%$ aqueous TBA solution were added; and the solution was made up to $4 \mathrm{~mL}$ with distilled water (d.w.). The solution was heated on boiling water bath for $60 \mathrm{~min}$ and cooled and $1 \mathrm{~mL}$ of d.w was added. $5 \mathrm{~mL}$ butanol and 
pyridine (15:1) was added and then centrifuged at $4000 \mathrm{rpm}$ for $10 \mathrm{~min}$. The absorbance of the orange layer was read at $532 \mathrm{~nm}$.

\section{Measurement of protein oxidation}

PC content was measured according to the procedure of Levine et al. ${ }^{[12]}$ Briefly, $100 \mu \mathrm{L}$ of supernatant was incubated with $0.5 \mathrm{~mL}$ of $10 \mathrm{mM}$ 2,4-dinitrophenylhydrazine (DNPH) in $2 \mathrm{M} \mathrm{HCl}$ for $60 \mathrm{~min}$ in the dark. Protein was precipitated using $0.5 \mathrm{~mL}$ of $20 \%$ Trichloroacetic acid (TCA) and then centrifuged at $10,000 \times g$ for $3 \mathrm{~min}$ at $4^{\circ} \mathrm{C}$. The supernatant was discarded and the pellet was washed with 1:1 ethyl acetate/ethanol twice by centrifugation $(3400 \times g$ for $5 \mathrm{~min}$ ) to remove DNPH. The pellet was dissolved after washing in $1.5 \mathrm{~mL}$ of $6 \mathrm{M}$ guanidine hydrochloride in phosphate buffer ( $\mathrm{pH}$ 6.5). Absorption was read at $370 \mathrm{~nm}$ in a spectrophotometer (Model SL 159, ELICO). The concentration of carbonyl groups was determined from the extinction coefficient of 22,000 $\mathrm{Mcm}^{-1}$ and expressed as nmol/mg protein.

\section{Measurement of superoxide radical}

SOR levels were measured according to the method of Das et al. ${ }^{[13]}$ Briefly, $200 \mu \mathrm{L}$ of homogenate was incubated with $80 \mu \mathrm{L}$ of $0.1 \%$ NBT in an oscillating water bath for $1 \mathrm{~h}$ at $37^{\circ} \mathrm{C}$. Termination of the assay and extraction of the reduced Nitroblue tetrazolium (NBT) were carried out by centrifuging the samples for $10 \mathrm{~min}$ at $200 \times g$ and then resuspending the pellets with $1 \mathrm{~mL}$ of glacial acetic acid. The absorbance was measured at $560 \mathrm{~nm}$ and converted to $\mu \mathrm{mol}$ diformazan using a standard curve generated from nitroblue diformazan. Final results were expressed as $\mu \mathrm{mol}$ diformazan/mg protein.

\section{Determination of total thiol, protein thiol, and nonprotein thiol levels}

The SH groups were determined according to the procedure of Sedlak and Lindsay ${ }^{[14]}$ For total thiol (T-SH), briefly, aliquots of $250 \mu \mathrm{L}$ of the homogenate were mixed in $5 \mathrm{~mL}$ test tubes with $750 \mu \mathrm{L}$ of $0.2 \mathrm{M}$ Tris buffer ( $\mathrm{pH} 8.2$ ) and $50 \mu \mathrm{L}$ of $0.01 \mathrm{M}$ DTNB. The mixture was made up to $5 \mathrm{~mL}$ with $3950 \mu \mathrm{L}$ of absolute methanol. A reagent blank and a sample blank were prepared in the same manner. The reaction mixture was centrifuged at $3000 \times g$ for $15 \mathrm{~min}$, and absorbance of the supernatants was read in a spectrophotometer at $412 \mathrm{~nm}$. Molar extinction coefficient at $412 \mathrm{~nm}$ was $13,100 / \mathrm{M} / \mathrm{cm}$ in both T-SH and nonprotein thiol (NP-SH) procedures.

For NP-SH, aliquots of $250 \mu \mathrm{L}$ of the homogenates were mixed in $5 \mathrm{~mL}$ test tubes with $200 \mu \mathrm{L}$ d.w. and $50 \mu \mathrm{L}$ of $50 \%$ TCA. The tubes were shaken intermittently for $10 \mathrm{~min}$ and centrifuged at $3000 \times g$ for $15 \mathrm{~min} .200 \mu \mathrm{L}$ of the supernatant was mixed with $400 \mu \mathrm{L}$ of $0.4 \mathrm{M}$ Tris buffer $(\mathrm{pH} 8.9)$. $10 \mu \mathrm{L}$ DTNB was then added, and absorbance was read within $5 \mathrm{~min}$ at $412 \mathrm{~nm}$ against a reagent blank. The protein thiol (P-SH) groups were calculated by subtracting the NP-SH from T-SH.

\section{Antioxidant enzymes activities}

Superoxide dismutase (SOD) activity was determined according to the method of Misra and Fridovich. ${ }^{[15]}$ Briefly, $100 \mu \mathrm{L}$ of the supernatant was added to $880 \mu \mathrm{L}$ of carbonate buffer $(0.05 \mathrm{M}, \mathrm{pH} 10.2)$ and $0.1 \mathrm{mM}$ ethylenediaminetetraacetic acid. $20 \mu \mathrm{L}$ of $30 \mathrm{mM}$ epinephrine in $0.05 \%$ acetic acid was added to the mixture, and absorbance was followed for $5 \mathrm{~min}$ at $480 \mathrm{~nm}$ in a spectrophotometer. The amount of enzyme that resulted in $50 \%$ inhibition of epinephrine auto-oxidation was defined as one unit.

Catalase (CAT) activity was measured according to the method of Aebi.

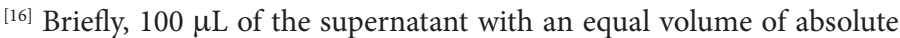
alcohol was incubated for $30 \mathrm{~min}$ at $0^{\circ} \mathrm{C}-8^{\circ} \mathrm{C}$ following which $10 \mu \mathrm{L}$ of Triton X-100 was added. A known volume of this was taken in an equal volume of $0.066 \mathrm{M}$ hydrogen peroxide $\left(\mathrm{H}_{2} \mathrm{O}_{2}\right)$ in phosphate buffer, and the decrease in absorbance was measured at $240 \mathrm{~nm}$ for a min in a spectrophotometer. An extinction coefficient of $43.6 \mathrm{Mcm}^{1}$ was used to determine enzyme activity, one unit of which was equal to $\mu \mathrm{mol}$ of $\mathrm{H}_{2} \mathrm{O}_{2}$ degraded $/ \mathrm{min} / \mathrm{mg}$ protein.

Glutathione peroxidase (GPx) activity was measured following the method of Flohé and Günzler. ${ }^{[17]}$ Briefly, the reaction mixture consisted of $500 \mu \mathrm{L}$ of phosphate buffer, $100 \mu \mathrm{L}$ of $0.01 \mathrm{M} \mathrm{GSH}, 100 \mu \mathrm{L}$ of $1.5 \mathrm{mM}$ $\mathrm{NADPH}$, and $100 \mu \mathrm{L}$ of glutathione reductase. $100 \mu \mathrm{L}$ of supernatant was added to the reaction mixture and incubated at $37^{\circ} \mathrm{C}$ for $10 \mathrm{~min} .50 \mu \mathrm{L}$ of $12 \mathrm{mM}$ t-butyl hydroperoxide was added to $450 \mu \mathrm{L}$ of cell extract reaction mixture and measured at $340 \mathrm{~nm}$ for $180 \mathrm{~s}$ in a spectrophotometer. Molar absorptivity of $6.22 \times 10^{3} \mathrm{M} / \mathrm{cm}$ was used to determine enzyme activity. One unit of activity is equal to $\mathrm{mM}$ NADPH oxidized $/ \mathrm{min} / \mathrm{mg}$ protein.

\section{Histopathology}

The LV and RV tissues were fixed in $10 \%$ buffered neutral formalin solution. After fixation, the tissues were processed by embedding in paraffin and were sectioned, stained with hematoxylin and eosin, and examined under a light microscope.

\section{Statistical analysis}

All the data were represented as the mean \pm standard error. The changes in serum lipid profile, LDH, and CK-MB between the groups were analyzed by one-way ANOVA. The changes in antioxidant enzyme activities and markers of oxidative stress were analyzed by two-way ANOVA. Significant " $F$ " ratios between the group means were further subjected to Tukey's test. Probability $(P)$ values at $<0.05$ were considered statistically significant.

\section{RESULTS}

\section{Body weight, heart weight, and heart weight/body weight ratio}

As represented in Table 1, no significant changes in BWs were noticed in ISO-administered rats when compared to their respective controls. $D h+$ ISO group exhibited a significant decrease in BWs over the ISO group. The heart weight (HW) and HW/BW ratio were recorded as an index of cardiac hypertrophy and were significantly increased in ISO-induced rats with respect to the normal control rats. A significant decrease in the HW and HW/BW ratio was evident in the supplemented

Table 1: Effect of ellagic acid, 4-hydroxy isophthalic acid, and Decalepis hamiltonii extract on body weight, heart weight, and heart weight to body weight ratio in isoproterenol-treated rats

\begin{tabular}{lccc}
\hline Groups & BW & HW & BW/HW \\
\hline Control & $256 \pm 1.85$ & $0.67 \pm 0.01$ & $0.26 \pm 0.01$ \\
ISO & $252 \pm 0.04$ & $0.99 \pm 0.02^{*}$ & $0.39 \pm 0.01^{*}$ \\
EA & $250 \pm 2.02$ & $0.65 \pm 0.01^{\#}$ & $0.25 \pm 0.03^{\#}$ \\
4-HIA & $252 \pm 2.84$ & $0.64 \pm 0.01^{\#}$ & $0.25 \pm 0.03^{\#}$ \\
Dh & $248 \pm 1.20$ & $0.62 \pm 0.01^{\#}$ & $0.24 \pm 0.03^{\#}$ \\
EA + ISO & $248 \pm 1.45$ & $0.90 \pm 0.05$ & $0.35 \pm 0.02$ \\
4-HIA + ISO & $249 \pm 2.08$ & $0.92 \pm 0.01^{\#}$ & $0.36 \pm 0.01$ \\
Dh + ISO & $245 \pm 0.33$ & $0.85 \pm 0.01^{\#}$ & $0.36 \pm 0.01^{\#}$ \\
\hline
\end{tabular}

Data are expressed as mean \pm SE of five animals/group and analyzed by one-way ANOVA followed by Tukey's test. *Significance between the sedentary control and ISO-treated group; "Comparison of ISO-treated group with other experimental groups; ${ }^{*}, P<0.05$ were considered significant. BW: Body weight; HW: Heart weight; ISO: Isoproterenol; EA: Ellagic acid; 4-HIA: 4-hydroxy isophthalic acid; Dh: Decalepis hamiltonii; SE: Standard error; LDH: Lactate dehydrogenase; CK-MB: Creatine kinase-MB 
ANUPAMA SINDHAGHATTA KARIYAPPA, et al.: Cardioprotective Effects of Decalepis hamiltonii and its Bioactive Compounds

rats over the ISO alone-treated rats. However, pretreatment of $D h$ extract to ISO-administered rats significantly reduced the $\mathrm{HW}$ and $\mathrm{HW} / \mathrm{BW}$ ratio by $14 \%$ and $8 \%$, respectively, over the ISO alone-treated rats.

\section{Serum lipid profile}

The serum lipid profile changes in the control and the ISO-administered rats are represented in Table 2. ISO-treated rats showed a significant increase in TC, TG, and LDL with a concomitant decrease in HDL levels compared to control rats. Pretreatment of EA, 4-HIA, and Dh extract to ISO-treated rats reduced the serum lipid profile changes compared to ISO alone-treated rats. Maximum reduction was evident in $\mathrm{Dh}+\mathrm{ISO}$ group in TC, TG, LDL, and HDL levels. Supplementation of EA, 4-HIA, and $D h$ extract significantly decreased the lipid profile changes over the ISO-treated rats.

\section{Cardiac marker enzymes}

Table 3 depicts the effects of $D h$ extract and bioactive compounds on the activities of cardiac marker enzymes (LDH and CK-MB) in the normal and ISO-treated rats. There is a significant increase in the levels of $\mathrm{LDH}(51 \%)$ and $\mathrm{CK}-\mathrm{MB}(50 \%)$ in the ISO-treated rats relative to the control rats. However, pretreatment of EA, 4-HIA, and Dh extract in ISO-administered rats reduced the serum levels of these enzymes. Pretreatment of $D h$ extract and the compounds significantly decreased the levels of these enzymes to the normal levels.

\section{Antioxidant enzyme activities}

The antioxidant enzyme activities were significantly decreased in the ISO-treated rats as represented in Figure 1. Region-specific changes were evident for SOD and CAT activities. Dh + ISO group exhibited higher SOD activity by $41 \%$ and $40 \%$ (LV and RV) compared to ISO alone-treated rats. A significant increase in CAT (LV - 57\%, RV - 56\%) activity was noticed in supplemented rats treated with ISO. A notable feature of GPx activity

Table 2: Effect of ellagic acid, 4-hydroxy isophthalic acid, and Decalepis hamiltonii extract on changes on serum lipid profile in isoproterenol-treated rats

\begin{tabular}{lcccc}
\hline Groups & TC $(\mathrm{mg} / \mathrm{dl})$ & TG $(\mathrm{mg} / \mathrm{dl})$ & $\begin{array}{c}\mathrm{HDL} \\
(\mathrm{mg} / \mathrm{dl})\end{array}$ & $\begin{array}{c}\mathrm{LDL} \\
(\mathrm{mg} / \mathrm{dl})\end{array}$ \\
\hline Con & $84.26 \pm 2.44$ & $91.57 \pm 0.59$ & $38.42 \pm 1.99$ & $27.85 \pm 2.18$ \\
ISO & $135.95 \pm 2.62^{*}$ & $123.93 \pm 3.10^{*}$ & $25.48 \pm 2.27^{*}$ & $85.69 \pm 1.60^{*}$ \\
EA & $79.17 \pm 3.24^{*}$ & $84.33 \pm 2.48^{*}$ & $49.44 \pm 1.98$ & $14.17 \pm 2.45^{*}$ \\
4-HIA & $82.43 \pm 2.09^{*}$ & $85.76 \pm 2.54^{*}$ & $48.33 \pm 2.23^{*}$ & $16.51 \pm 3.10^{*}$ \\
Dh & $75.68 \pm 1.09^{*}$ & $79.48 \pm 0.75^{*}$ & $51.45 \pm 1.99^{*}$ & $8.25 \pm 1.22^{*}$ \\
EA + ISO & $109.26 \pm 1.27^{*}$ & $112.64 \pm 3.06^{*}$ & $41.98 \pm 1.78^{*}$ & $25.24 \pm 1.98^{*}$ \\
4-HIA + ISO & $113.46 \pm 5.25^{*}$ & $108.41 \pm 2.2$ & $39.21 \pm 2.36$ & $25.53 \pm 1.82^{*}$ \\
Dh + ISO & $96.06 \pm 1.59^{*}$ & $99.51 \pm 2.89^{*}$ & $44.17 \pm 3.16$ & $13.57 \pm 1.95^{*}$ \\
\hline
\end{tabular}

Values are expressed as mean \pm SE and were analyzed through one-way ANOVA followed by Tukey's test. ${ }^{*}$ Significance between the sedentary control and ISO-treated group; "Comparison of ISO-treated group with other experimental groups; ${ }^{*, *} P<0.05$ were considered significant. TC: Total cholesterol;

TG: Triglycerides; HDL: High-density lipoprotein; LDL: Low-density lipoprotein; ISO: Isoproterenol; EA: Ellagic acid; 4-HIA: 4-hydroxy isophthalic acid; Dh: Decalepis hamiltonii; SE: Standard error is that insignificant changes were noticed between the regions. However, activity was significantly enhanced only in EA + ISO (LV - 14\%, RV - 27\%) and $\mathrm{Dh}+\mathrm{ISO}$ (LV - 23\%, RV - 36\%) groups over the ISO alone-treated rats. Supplemented rats showed increased antioxidant enzyme activities compared to ISO alone-treated rats.

\section{Markers of oxidative stress}

The levels of oxidative stress markers (MDA and PC) were significantly elevated in the rats treated with ISO compared to the control rats. The MDA and PC contents were decreased maximally in the $D h+$ ISO-treated rats in LV (60\% and 51\%) and RV (65\% and 50\%) over the ISO alone-treated rats. Supplementation of the EA, 4-HIA, and Dh extract was found to be more effective in curtailing lipid peroxidation and protein oxidation [Figure $2 \mathrm{a}$ and $\mathrm{b}$ ]. However, insignificant changes were observed between the regions.

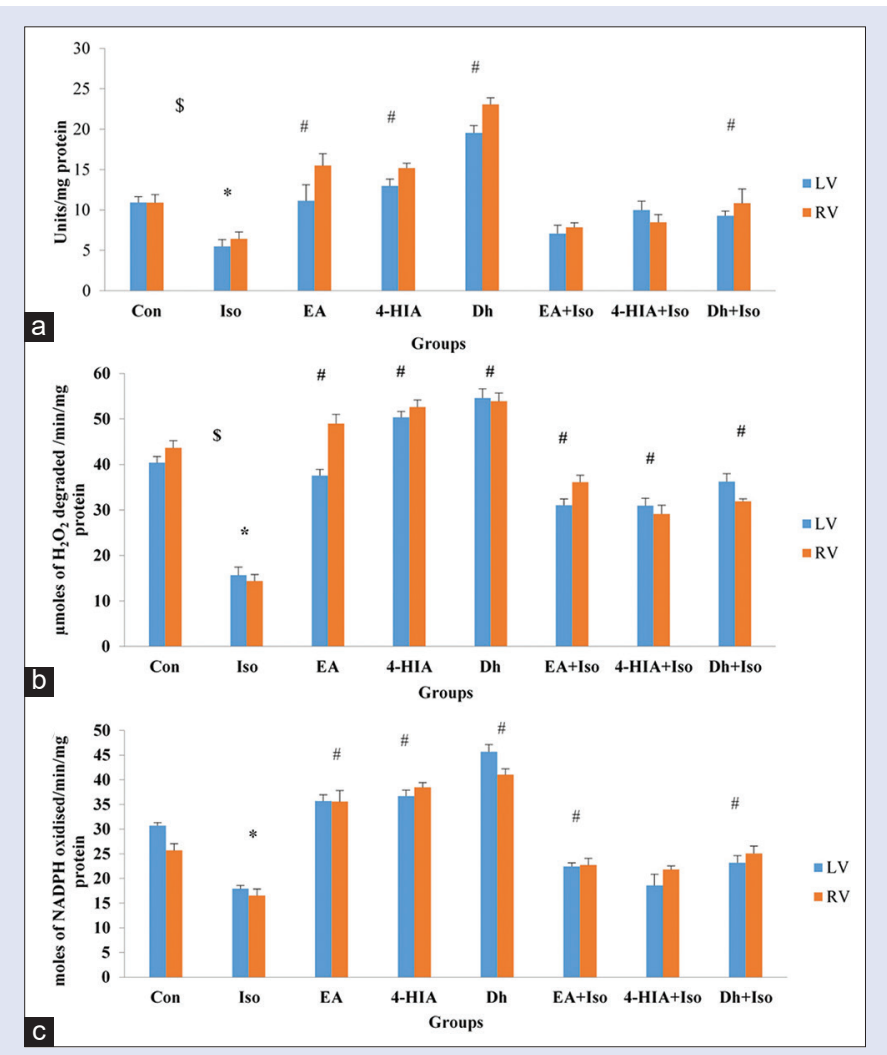

Figure 1: The levels of SOD (a), CAT (b) and GPx (c) in the control and experimental groups. The data are expressed as mean \pm SE from six independent animals and analyzed by two-way ANOVA followed by Tukey's test. *Significance between the control and ISO alone treated group. "Comparison of ISO alone treated the group with other experimental groups. ${ }^{5}$ indicates the significance between the regions. *\#S Probability values $P<0.05$ were considered significant. SOD: Superoxide dismutase; CAT: Catalase; GPx: Glutathione peroxidase; SE: Standard error; ISO: Isoproterenol

Table 3: Effect of ellagic acid, 4-hydroxy isophthalic acid, and Decalepis hamiltonii extract on lactate dehydrogenase and creatine kinase-MB activities in isoproterenol-treated rats

\begin{tabular}{|c|c|c|c|c|c|c|c|c|}
\hline Groups & Con & ISO & EA & 4-HIA & Dh & $E A+I S O$ & 4-HIA + ISO & Dh + ISO \\
\hline $\mathrm{LDH}$ & $95.14 \pm 2.75$ & $193.6 \pm 2.49^{*}$ & $95.84 \pm 1.71^{\#}$ & $95.47 \pm 2.39^{\#}$ & $85.63 \pm 3.24^{\#}$ & $156.03 \pm 2.13^{\#}$ & $166.59 \pm 3.17^{\#}$ & $123.46 \pm 1.88^{\#}$ \\
\hline CK-MB & $85.17 \pm 2.65$ & $189.93 \pm 3.12^{*}$ & $75.71 \pm 1.69^{\#}$ & $80.56 \pm 2.33^{\#}$ & $59 \pm 1.75^{\#}$ & $142.95 \pm 3.48^{\#}$ & $151.58 \pm 2.75^{\#}$ & $133.26 \pm 2.02^{\#}$ \\
\hline
\end{tabular}

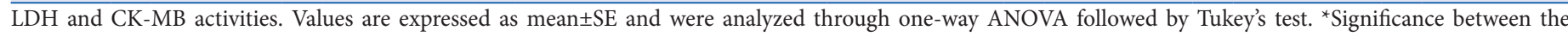

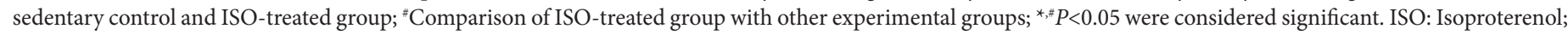
EA: Ellagic acid; 4-HIA: 4-hydroxy isophthalic acid; Dh: Decalepis hamiltonii; SE: Standard error; LDH: Lactate dehydrogenase; CK-MB: Creatine kinase-MB 
SOR levels were measured in the myocardium as it is one of the major free radicals produced during physiological reactions. The levels were significantly higher in the LV compared to RV in all the experimental groups. As reported in Figure 2c, there was a significant increase in SOR levels in the LV (47\%) and RV (42\%) in ISO alone-treated rats. Notably, both supplemented controls and supplemented rats administered with ISO showed reduced radical generation.

The T-SH, NP-SH, and P-SH levels exhibited insignificant changes between the regions. In ISO-administered rats, a significant decrease in the levels of SHs in LV (T-SH - 48\%, NP-SH - 52\%, P-SH - 44\%)

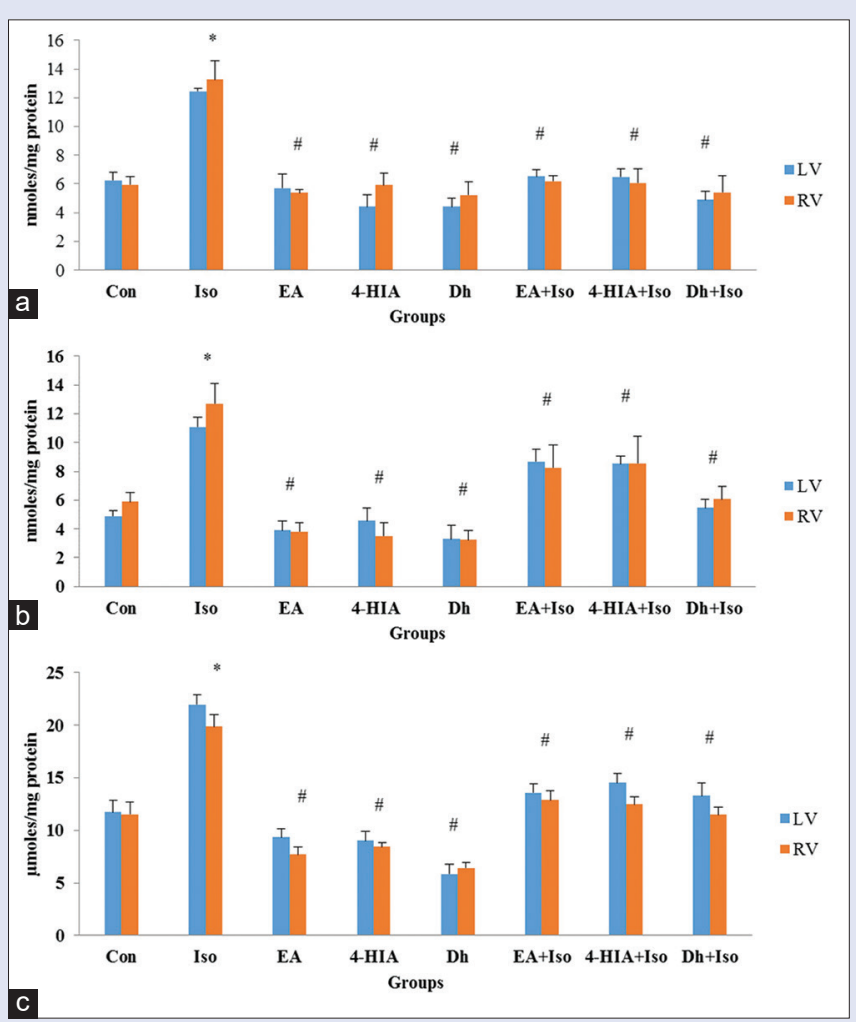

Figure 2: The levels of MDA (a), PC (b), and SOR (c) in the control and experimental groups. The data are expressed as mean \pm SE from six independent animals and analyzed by two-way ANOVA followed by Tukey's test. *Significance between the control and ISO alone treated group. "Comparison of ISO alone-treated group with other experimental groups. * Probability values $P<0.05$ were considered significant. MDA: Malondialdehyde; PC: Protein carbonyl; SOR: Superoxide radical; SE: Standard error and RV (T-SH - 60\%, NP-SH - 26\%, P-SH - 81\%) was observed when compared to the controls. T-SH levels were significantly enhanced in the $\mathrm{Dh}+\mathrm{ISO}$-treated and EA + ISO-treated rats. However, NP-SH levels were significantly upregulated only in $\mathrm{Dh}+\mathrm{ISO}$-treated rats. A remarkable aspect of the P-SH levels is that insignificant changes were noticed in the supplemented groups treated with ISO. Pretreatment of EA, 4-HIA, and Dh extract increased the levels of T-SH, NP-SH, and $\mathrm{P}-\mathrm{SH}$ in comparison with ISO alone-treated rats [Table 4].

\section{Histopathological changes}

The histopathology of the LV and RV regions of the control rats showed normal myocardial architecture with no evidence of necrosis and inflammation. However, extensive membrane damage, focal myocardial necrosis, and infiltration of the inflammatory cells were observed in both the regions of ISO alone-treated rats. Pretreatment with $D h$ extract offered better protection compared to EA and 4-HIA against the deleterious effects of ISO in terms of lesser extent of infiltration and myonecrosis. Supplemented groups revealed normal myocardial histoarchitecture [Figures 3 and 4].

\section{DISCUSSION}

Cardiac diseases have been linked to oxidative stress, which is due to the reactions of free radicals with proteins, lipids, and DNA. Antioxidants from plant sources have been considered as an effective treatment for cardiovascular diseases. The root extract of $D h$ is consumed for various health benefits and is a cocktail of many antioxidant compounds. ${ }^{[6]}$ Studies have reported that aqueous root extract has protective effects against neurotoxicity and age-related oxidative stress. ${ }^{[8]}$ Therefore, the present study was undertaken to investigate the cardioprotective potential of $D h$ extract and its bioactive compounds in ameliorating ISO-induced $\mathrm{MI}$ in rats. $\mathrm{LV}$ and RV regions were selected due to the differences in morphology and function as well as in the distribution of antioxidant defense systems.

In our study, BW changes were insignificant among the experimental groups and are in line with previous reports. ${ }^{[18]}$ However, we observed a significant increase in HW and HW/BW ratio in ISO alone-treated rats indicating cardiac hypertrophy. Several studies also reported that increased HW and HW/BW ratio in ISO-induced myocardial-infarcted rats might be due to edematous intramuscular space, increased protein and water content, necrosis, and penetration of inflammatory cells to damaged cardiac tissues. ${ }^{[10,19]}$ Supplementation of $D h$ extract significantly reduced the HW and HW/BW ratio, thereby attenuating cardiac hypertrophy in ISO-administered rats.

Hyperlipidemia is one of the leading causes of cardiovascular diseases. Altered lipid metabolism plays an important role in the development of atherosclerosis and in the progression of $\mathrm{MI}^{[20]}$ In the present

Table 4: Effect of ellagic acid, 4-hydroxy isophthalic acid and Decalepis hamiltonii extract on total thiol, nonprotein thiols, protein thiol activities in isoproterenol-treated rats

\begin{tabular}{|c|c|c|c|c|c|c|c|c|c|}
\hline \multirow[t]{2}{*}{ Parameters } & \multirow[t]{2}{*}{ Regions } & \multicolumn{7}{|c|}{ Groups } & \multirow[t]{2}{*}{$\mathrm{Dh}+\mathrm{ISO}$} \\
\hline & & CON & ISO & EA & 4-HIA & $\mathrm{Dh}$ & $E A+I S O$ & 4-HIA + ISO & \\
\hline \multirow[t]{2}{*}{ T-SH } & LV & $234.59 \pm 8.0$ & $121.62 \pm 7.67^{*}$ & $368.31 \pm 6.56^{\#}$ & $293.65 \pm 12.02^{*}$ & $394.91 \pm 5.06^{\#}$ & $146.47 \pm 5.36^{*}$ & $131.43 \pm 9.74$ & $174.97 \pm 5.49^{*}$ \\
\hline & RV & $247.98 \pm 12.4$ & $98.53 \pm 4.77$ & $354.46 \pm 6.42$ & $312.32 \pm 6.55$ & $413.91 \pm 5.75$ & $138.77 \pm 6.23$ & $114.41 \pm 6.15$ & $163.42 \pm 5.00$ \\
\hline \multirow[t]{2}{*}{ NP-SH } & LV & $140.1 \pm 9.54$ & $68.64 \pm 8.49^{*}$ & $132.01 \pm 9.17^{\#}$ & $166.39 \pm 9.52^{\#}$ & $181.75 \pm 11.23^{\#}$ & $76.96 \pm 5.28$ & $62.62 \pm 5.96$ & $99.54 \pm 9.45^{\#}$ \\
\hline & RV & $95.40 \pm 7.91$ & $69.90 \pm 9.54$ & $128.41 \pm 12.29$ & $147.18 \pm 11.65$ & $229.93 \pm 15.00$ & $56.59 \pm 8.36$ & $55.81 \pm 8.44$ & $111.98 \pm 14.89$ \\
\hline \multirow[t]{2}{*}{ P-SH } & LV & $94.49 \pm 4.56$ & $52.97 \pm 8.87^{*}$ & $236.30 \pm 15.73^{\#}$ & $127.26 \pm 15.32^{\#}$ & $213.15 \pm 11.49^{\#}$ & $69.51 \pm 9.76$ & $65.14 \pm 11.02$ & $64.77 \pm 10.89$ \\
\hline & RV & $152.58 \pm 11.20$ & $28.62 \pm 10.72$ & $226.05 \pm 7.80$ & $165.14 \pm 10.31$ & $183.98 \pm 16.49$ & $82.18 \pm 13.60$ & $58.59 \pm 14.55$ & $64.78 \pm 10.54$ \\
\hline
\end{tabular}

T-SH: Total thiols; NP-SH: Nonprotein thiols; P-SH: Protein thiols. Values are expressed as mean \pm SE and were analyzed through two-way ANOVA followed by Tukey's test. ${ }^{*}$ Significance between the sedentary control and ISO-treated group; ${ }^{*}$ Comparison of ISO-treated group with other experimental groups; ${ }^{*, *} P<0.05$ were considered significant. ISO: Isoproterenol; EA: Ellagic acid; 4-HIA: 4-hydroxy isophthalic acid; Dh: Decalepis hamiltonii; SE: Standard error; LV: Left ventricle; RV: Right ventricle 
ANUPAMA SINDHAGHATTA KARIYAPPA, et al.: Cardioprotective Effects of Decalepis hamiltonii and its Bioactive Compounds

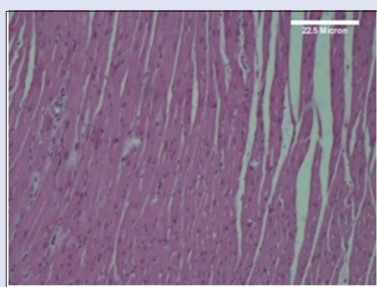

Control

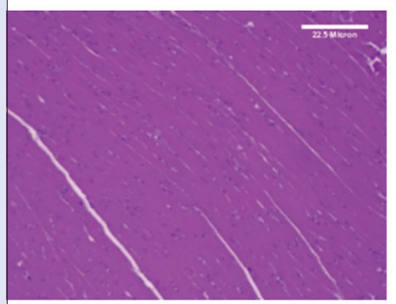

EA

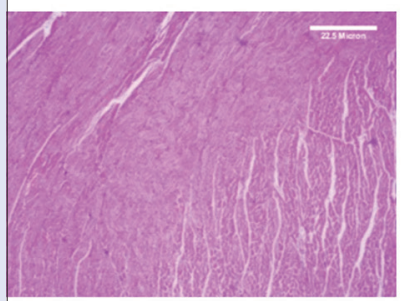

$\mathrm{Dh}$

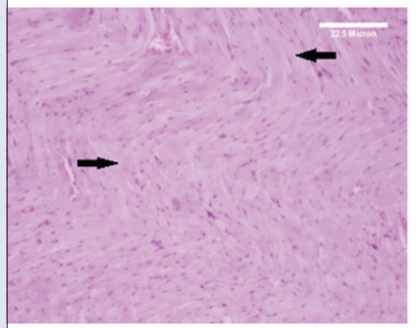

4-HIA+ISO

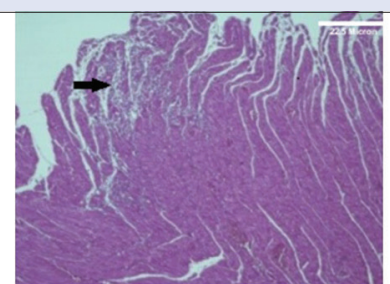

ISO

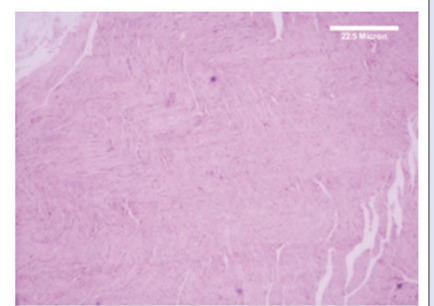

4-HIA

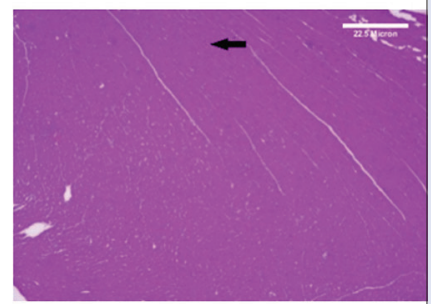

EA+ISO

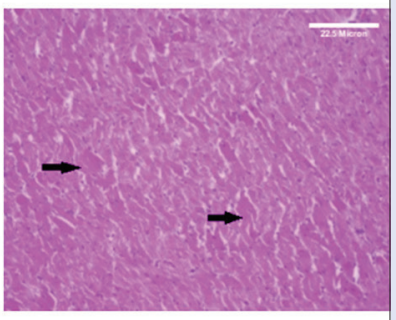

$\mathrm{Dh}+\mathrm{ISO}$
Figure 3: Photomicrographs of histopathological changes of $L V$ of control, supplemented, and ISO-treated rats. Control, ISO, EA, 4-HIA, Decalepis hamiltonii extract, EA + ISO, 4-HIA + ISO, and Decalepis hamiltonii + ISO. Tissues were stained with hematoxylin and eosin, visualized under a light microscope at $\times 100$. Arrow indicates infiltration of inflammatory cells and myocardial necrosis. LV: Left ventricle; ISO: Isoproterenol; EA: Ellagic acid; 4-HIA: 4-hydroxy isophthalic acid

study, ISO administration significantly elevated lipid profile markers and decreased HDL levels. This may be attributed to enhanced lipid biosynthesis by cyclic-AMP and decreased activity of lipoprotein lipase in the myocardium. Our results are consistent with the previous studies of Abbas. ${ }^{[21]}$ Pretreatment with EA, 4-HIA, and Dh extract improved ISO-induced hyperlipidemic damage, suggesting their lipid-lowering effects. Several reports have shown that pretreatment with plant extracts and natural compounds prevent ISO-induced hyperlipidemia. ${ }^{[22,23]}$

$\mathrm{LDH}$ and $\mathrm{CK}-\mathrm{MB}$ are the reliable diagnostic markers to assess the severity of ISO-induced MI. ${ }^{[24]}$ These marker enzymes were elevated in the serum following ISO treatment, which indicates damage to myocardial tissues. Our findings on increased levels of these enzymes are similar to the earlier reports. ${ }^{[25]}$ Administration of EA, 4-HIA, and Dh extract with ISO

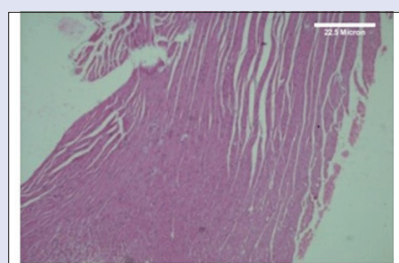

Control

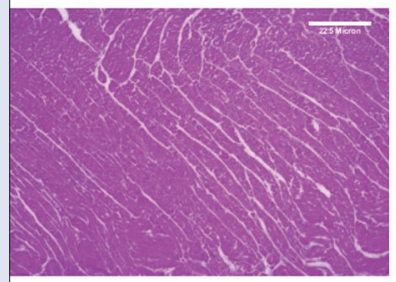

EA

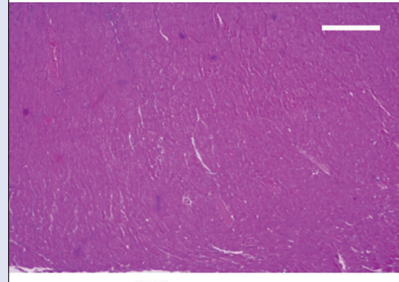

$\mathrm{Dh}$

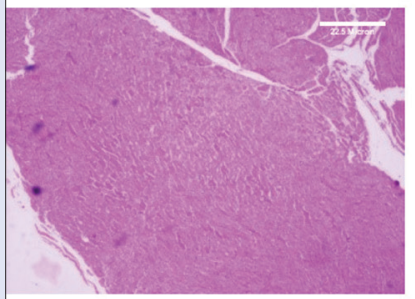

4-HIA+ISO

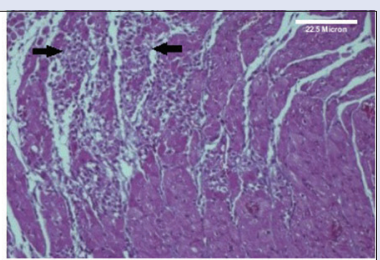

ISO

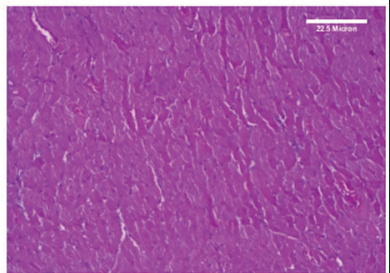

4-HIA

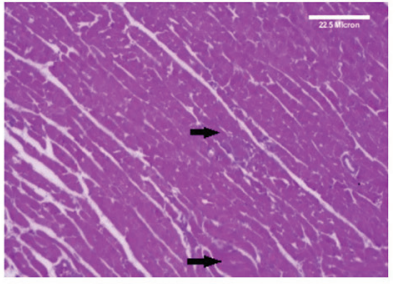

$\mathrm{EA}+\mathrm{ISO}$

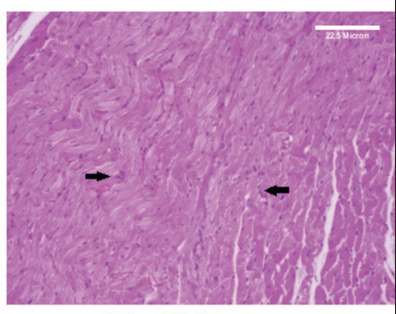

$\mathrm{Dh}+\mathrm{ISO}$
Figure 4: Photomicrographs of histopathological changes of RV of control, supplemented, and ISO-treated rats. Control, ISO, EA, 4-HIA, Decalepis hamiltonii extract, EA + ISO, 4-HIA + ISO, and Decalepis hamiltonii + ISO. Tissues were stained with hematoxylin and eosin, visualized under a light microscope at $\times 100$. Arrow indicates infiltration of inflammatory cells and myocardial necrosis. RV: Right ventricle; ISO: Isoproterenol; EA: Ellagic acid; 4-HIA: 4-hydroxy isophthalic acid

resulted in a significant decrease in the concentration of these enzymes into the circulation which could be due to antioxidant and free radical scavenging activities of the compounds and the extract, suggesting that Dh extract and its compounds maintained the membrane integrity, thereby reducing the leakage of these enzymes.

Oxidative stress is considered to be one of the mechanisms of ISO-induced MI. Antioxidants constitute the first line of cellular defense system that limits the toxicity associated with free radicals. SOD, CAT, and GPx are the important antioxidant defense enzymes involved in the dismutation of SORs and $\mathrm{H}_{2} \mathrm{O}_{2}$. ISO treatment resulted in decreased activities of these antioxidant enzymes, indicating severe oxidative damage. The results of the present study are in line with previous reports. ${ }^{[26]} \mathrm{RV}$ exhibited higher antioxidant enzyme activities compared to the LV may be due to the structural differences and enhanced free radical scavenging activity. ${ }^{[27]}$ Pretreatment of EA, 4-HIA, and Dh extract significantly upregulated the activity of these enzymes compared to the ISO alone-induced rats. The increased activity in the supplemented rats may be attributed to the modulation of nuclear factors, such as Nrf2, activator protein-1, and 
$\mathrm{NF \kappa B}$, which in turn bind to the antioxidant response elements in the regulatory region of the target genes and induces the transcription of antioxidant enzymes. ${ }^{[28]}$ Previous studies from our laboratory and others have demonstrated that supplementation of $D h$ extract and EA improves the antioxidant status in the different regions of the brain. ${ }^{[8,29]}$

Lipid peroxidation is an important pathological event in myocardial necrosis, leading to the accumulation of lipid hydroperoxides, causing irreversible damage to the myocardial membrane. ${ }^{[30]}$ In the present study, MDA levels were significantly increased in the ISO alone-treated rats, which indicates enhanced ROS generation by auto-oxidation of ISO, thereby initiating lipid peroxidation. ${ }^{[31]}$ Earlier reports also showed elevated MDA levels in ISO alone-treated rats. ${ }^{[24]}$ Proteins are also prone to oxidation by free radicals attacking amino acid side chains to produce carbonyl groups. ${ }^{[32]}$ Our results indicate higher oxidation of proteins in the ISO alone-treated rats, suggesting severe damage to the myocardium. However, pretreatment of EA, 4-HIA, and Dh extract resulted in reduced levels of MDA and PC in ISO-treated rats, indicating that the compounds and extract possess antilipid peroxidative and antiprotein oxidation properties. Previous studies have also demonstrated that supplementation of $D h$ extract and its bioactive compounds decreased the lipid peroxidation and protein oxidation products in the rat liver and brain. ${ }^{[33,34]}$

SOR is one of the major free radicals generated during oxidative phosphorylation found to higher in the LV compared to RV. Increased radical generation in ISO alone-treated rats is attributed to the oxidation of ISO, causing the cardiac tissues more prone to oxidative damage. This is in accordance with the previous reports of Dhivya et al., ${ }^{[35]}$ where increased ROS generation was observed in ISO-induced MI in rats. The SOR levels were significantly reduced in the supplemented as well as ISO-treated groups. This modulatory effect of $D h$ extract and its constituents could be due to scavenging of free radicals, thus increasing SOD activity as evident in the supplemented groups.

$\mathrm{SH}$ levels were determined as they are the main constituents of intracellular nonprotein sulfhydryl groups involved in many cellular functions, such as drug metabolism and detoxification of free radicals. ${ }^{[36]}$ In the present study, a significant decrease in the levels of SHs was observed in the ISO alone-treated rats, indicating decline in the antioxidant status in the myocardium. Decreased SH levels may be due to its increased utilization to enhance the activity of GPx. Administration of $D h$ extract and its bioactive compounds significantly elevated the levels of SHs in the ISO-treated rats by maintaining the $\mathrm{SH}$ groups in the reduced state and enhancing the antioxidant status. ${ }^{[37]}$ Protective effects of EA, 4-HIA, and Dh extract on myocardial histoarchitecture were further confirmed by light microscopic studies. Histopathological examination of LV and RV regions of ISO alone-treated rats showed edema, infiltration, and myocardial necrosis. Several studies have reported similar histopathological changes in the ISO-treated rats. ${ }^{[38]}$ These changes may be due to increased oxidative stress and alterations in lipid metabolism, thereby causing the structural modifications. However, pretreatment with $\mathrm{Dh}$ extract followed by ISO administration exhibited improved myocardial architecture in terms of mild necrosis with the presence of some inflammatory cells compared to ISO per se-administered rats. These results further confirmed the cardioprotective potential of $D h$ extract.

\section{CONCLUSION}

The present study revealed the cardioprotective potential of $D h$ extract and its bioactive compounds against ISO-induced MI by modulating cardiac marker enzymes, lipid profile, and antioxidant status. The $D h$ extract was found to be more effective than individual bioactive compounds. However, further studies are warranted to establish the modulation of NFKB and Nrf2 signaling pathways by $D h$ extract to evaluate its protective effect in treating cardiovascular diseases.

\section{Financial support and sponsorship}

This work was supported by the financial assistance from University Grants Commission-Basic Scientific Research (U.O.No.DEV: DU2G: C-BSR: MB and BT: 2012-13). We wish to thank the Department of Microbiology and Biotechnology, Bangalore University, Bengaluru, for providing necessary infrastructural facilities.

\section{Conflicts of interest}

There are no conflicts of interest.

\section{REFERENCES}

1. Ashokkumar R, Jamuna S, Sakeena Sadullah MS, Niranjali Devaraj S. Vitexin protects isoproterenol induced post myocardial injury by modulating hipposignaling and ER stress responses. Biochem Biophys Res Commun 2018;496:731-7.

2. Wang Z, Zhang J, Ren T, Dong Z. Targeted metabolomic profiling of cardioprotective effect of Ginkgo biloba L. Extract on myocardial ischemia in rats. Phytomedicine 2016;23:621-31.

3. Rajadurai M, Prince PS. Preventive effect of naringin on isoproterenol-induced cardiotoxicity in Wistar rats: An in vivo and in vitro study. Toxicology 2007;232:216-25.

4. Kumar M, Kasala ER, Bodduluru LN, Kumar V, Lahkar M. Molecular and biochemical evidence on the protective effects of quercetin in isoproterenol-induced acute myocardial injury in rats. J Biochem Mol Toxicol 2017;31:1-8.

5. Srivastava A, Rao LJ, Shivanandappa T. Isolation of ellagic acid from the aqueous extract of the roots of Decalepis hamiltonii: Antioxidant activity and cytoprotective effect. Food Chem 2007; 103:224-33.

6. Srivastava A, Harish R, Shivanandappa T. Novel antioxidant compounds from the aqueous extract of the roots of Decalepis hamiltonii (Wight and Arn.) and their inhibitory effect on low-density lipoprotein oxidation. J Agric Food Chem 2006;54:790-5.

7. Naveen S, Khanum F. Antidiabetic, antiatherosclerotic and hepatoprotective Properties of Decalepis hamiltonii in streptozotocin-induced diabetic rats. J Food Biochem 2010;34:1231-48

8. Ravikiran T, Sowbhagya R, Anupama SK, Anand S, Bhagyalakshmi D. Age-related changes in the brain antioxidant status: Modulation by dietary supplementation of Decalepis hamiltonit and physical exercise. Mol Cell Biochem 2016;419:103-13.

9. Ebenezar KK, Sathish V, Devaki T. Effect of arginine and lysine on mitochondrial function during isoproterenol induced myocardial infarction in rats. Nutr Res 2003;23:1417-25.

10. Al-Rasheed NM, Al-Rasheed NM, Al-Rabeeah DA, Al-Barrak HS, Al-Salman SA, Ibrahim SA, et al. Possible protective mechanisms exerted by metformin or metformin and Vitamin $E$ in isoproterenol-induced cardiac injury. J Cell Biochem 2018;119:3903-12.

11. Ohkawa $\mathrm{H}$, Ohishi $\mathrm{N}$, Yagi K. Assay for lipid peroxides in animal tissues by thiobarbituric acid reaction. Anal Biochem 1979;95:351-8.

12. Levine RL, Williams JA, Stadtman ER, Shacter E. Carbonyl assays for determination of oxidatively modified proteins. Methods Enzymol 1994;233:346-57.

13. Das UN, Padma M, Sagar PS, Ramesh G, Koratkar R. Stimulation of free radical generation in human leukocytes by various agents including tumor necrosis factor is a calmodulin dependent process. Biochem Biophys Res Commun 1990;167:1030-6.

14. Sedlak J, Lindsay RH. Estimation of total, protein-bound, and nonprotein sulfhydryl groups in tissue with Ellman's reagent. Anal Biochem 1968;25:192-205.

15. Misra HP, Fridovich I. The role of superoxide anion in the autoxidation of epinephrine and a simple assay for superoxide dismutase. J Biol Chem 1972;247:3170-5

16. Aebi H. Catalase in vitro. Methods Enzymol 1984;105:121-6.

17. Flohé L, Günzler WA. Assays of glutathione peroxidase. Methods Enzymol 1984;105:114-21.

18. Imran A, Butt MS, Arshad MS, Arshad MU, Saeed F, Sohaib M, et al. Exploring the potential of black tea based flavonoids against hyperlipidemia related disorders. Lipids Health Dis 2018;17:57.

19. Saranya S, Padma VV. Berbamine protects the heart from isoproterenol induced myocardial infarction by modulating eNOS and iNOS expressions in rats. J Appl Biomed 2018;16:301-10.

20. Abhilash PA, Nisha P, Prathapan A, Nampoothiri SV, Lijo Cherian O, Sunitha TK, et al Cardioprotective effects of aqueous extract of Oxalis corniculata in experimental myocardial infarction. Exp Toxicol Pathol 2011;63:535-40

21. Abbas AM. Cardioprotective effect of resveratrol analogue isorhapontigenin versus 
ANUPAMA SINDHAGHATTA KARIYAPPA, et al.: Cardioprotective Effects of Decalepis hamiltonii and its Bioactive Compounds

omega-3 fatty acids in isoproterenol-induced myocardial infarction in rats. J Physiol Biochem 2016;72:469-84

22. Jiang $Y$, Dai $M$, Nie WJ, Yang $X R$, Zeng $X C$. Effects of the ethanol extract of black mulberry (Morus nigra L.) fruit on experimental atherosclerosis in rats. J Ethnopharmacol 2017;200:228-35

23. Wong ZW, Thanikachalam PV, Ramamurthy S. Molecular understanding of the protective role of natural products on isoproterenol-induced myocardial infarction: A review. Biomed Pharmacother 2017;94:1145-66.

24. Sunmonu TO, Afolayan AJ. Protective effect of Artemisia afra Jacq. On isoproterenol-induced myocardial injury in Wistar rats. Food Chem Toxicol 2010;48:1969-72.

25. Akila P, Vennila L. Chlorogenic acid a dietary polyphenol attenuates isoproterenol induced myocardial oxidative stress in rat myocardium: An in vivo study. Biomed Pharmacother 2016;84:208-14

26. Khan V, Sharma S, Bhandari U, Ali SM, Haque SE. Raspberry ketone protects against isoproterenol-induced myocardial infarction in rats. Life Sci 2018;194:205-12.

27. Asha Devi S, Prathima S, Subramanyam MV. Dietary Vitamin E and physical exercise: II. Antioxidant status and lipofuscin-like substances in aging rat heart. Exp Geronto 2003;38:291-7.

28. Yuliani S, Mustofa, Partadiredja G. The neuroprotective effects of an ethanolic turmeric (Curcuma longa L.) extract against trimethyltin-induced oxidative stress in rats. Nutr Neurosci 2018;7:1-8.

29. Kannan MM, Quine SD. Ellagic acid protects mitochondria from beta-adrenergic agonist induced myocardial damage in rats; evidence from in vivo, in vitro and ultra structural study. Food Res Int 2012;45:1-8

30. Sahu BD, Putcha UK, Kuncha M, Rachamalla SS, Sistla R. Carnosic acid promotes myocardia antioxidant response and prevents isoproterenol-induced myocardial oxidative stress and apoptosis in mice. Mol Cell Biochem 2014:394:163-76.

31. Shahzad S, Mateen S, Mubeena Mariyath PM, Naeem SS, Akhtar K, Rizvi W, et al. Protective effect of syringaldehyde on biomolecular oxidation, inflammation and histopathological alterations in isoproterenol induced cardiotoxicity in rats. Biomed Pharmacother 2018; 108:625-33.

32. Evran B, Karpuzoğlu H, Develi S, Kalaz EB, Soluk-Tekkeșin M, Olgac V, et al. Effects of carnosine on prooxidant-antioxidant status in heart tissue, plasma and erythrocytes of rats with isoproterenol-induced myocardial infarction. Pharmacol Rep 2014;66:81-6.

33. Sowbhagya R, Anupama SK, Bhagyalakshmi D, Anand S, Ravikiran T. Modulatory effects of Decalepis hamiltonii extract and its compounds on the antioxidant status of the aging rat brain. J Pharm Bioallied Sci 2017:9:8-15

34. Srivastava A, Rao LJ, Shivanandappa T. A novel cytoprotective antioxidant: 4-hydroxy isophthalic acid. Food Chem 2012;132:1959-65.

35. Dhivya V, Priya LB, Chirayil HT, Sathiskumar S, Huang CY, Padma VV, et al. Piperine modulates isoproterenol induced myocardial ischemia through antioxidant and anti-dyslipidemic effect in male Wistar rats. Biomed Pharmacother 2017;87:705-13.

36. Amudha G, Josephine A, Mythili Y, Sundarapandiyan R, Varalakshmi P. Therapeutic efficacy of DL-alpha-lipoic acid on cyclosporine $A$ induced renal alterations. Eur $J$ Pharmacol 2007:571:209-14.

37. Binu P, Gifty K, Vineetha RC, Abhilash S, Arathi P, Nair RH. Eugenol, a plant-derived phenolic nutraceutical, protects thiol (SH) group in myocardium from ROS-mediated oxidation under chemotherapeutic stress induced by arsenic trioxide - A in vivo model study. Drug Chem Toxicol 2018:41:352-7.

38. Suchal K, Malik S, Gamad N, Malhotra RK, Goyal SN, Bhatia J, et al. Kampeferol protects against oxidative stress and apoptotic damage in experimental model of isoproterenol-induced cardiac toxicity in rats. Phytomedicine 2016:23:1401-8. 